\title{
BMJ Open Quality Improving end-of-life care for adults with cystic fibrosis: an improvement project
}

\author{
Fiona Cathcart (D) , ${ }^{1}$ Jayne Wood, ${ }^{2}$ Su Madge ${ }^{1}$
}

To cite: Cathcart F, Wood J, Madge S. Improving end-oflife care for adults with cystic fibrosis: an improvement project. BMJ Open Quality 2020;9:e000861. doi:10.1136/ bmjoq-2019-000861

Received 17 0ctober 2019 Revised 28 June 2020 Accepted 8 July 2020
Check for updates

(C) Author(s) (or their employer(s)) 2020. Re-use permitted under CC BY-NC. No commercial re-use. See rights and permissions. Published by BMJ.

${ }^{1}$ Adult Cystic Fibrosis, Royal Brompton and Harefield NHS Foundation Trust, London, UK ${ }^{2}$ Symptom Control and Palliative Care, Royal Marsden NHS Foundation Trust, London, UK

Correspondence to

Fiona Cathcart;

f.cathcar@@rbht.nhs.uk

\section{ABSTRACT}

Background Cystic fibrosis (CF) is a life-limiting disease that results in premature death mainly due to respiratory failure. Literature suggests that for many people with CF end-of-life wishes are discussed too late or not at all, with most dying in hospital. The aim of this project was to improve end-of-life care for adults with CF.

Design Three improvement cycles were carried out over a 2-year period in one of the largest adult CF centres in Europe. The first cycle involved implementing regular multidisciplinary team (MDT) debriefs after a patient death with increased education. The second cycle involved codesigning a CF-specific advance care plan (ACP) with patients, families, bereaved relatives and experts across the UK, then implementing this into our service. The final cycle was designing a CF-specific end of life, online course for clinicians. Success was measured by: use of ACP and whether patients had died in their preferred location, patient feedback via a survey and satisfaction with the online course using a postcourse report.

Results The number of patients given the opportunity to discuss their end of life wishes increased from $10 \%$ to $85 \%$. The number of patients who died in their preferred location increased from $7 \%$ to $85 \%$ over the 2 -year project time. Patient feedback has been overwhelmingly positive. The key barrier has been changing MDT culture, overcoming this required the engagement of the whole team. The online course has been successful with 258 participants to date from 26 countries.

Conclusion Education, staff support and a CF-specific ACP document empowered healthcare professionals to initiate difficult conversations to improve end-of-life care.

\section{PROBLEM}

Cystic fibrosis (CF) is an inherited disease with no cure, however, over the last decade, there have been significant advances in medical management. Previously described as a disease of childhood, death from CF is now rare in the under 19 year olds in the UK. ${ }^{1}$ Consequently, an increase of up to $75 \%$ in the number of adults with CF throughout Europe has been predicted by $2025 .{ }^{1}$ This inevitably means that healthcare professionals (HCP) providing $\mathrm{CF}$ and/or palliative care may start to see more adults with $\mathrm{CF}$ at end of life (EoL) in both specialist CF centres and the community. As the largest CF centre in the UK caring for approximately 560 adults with
CF (prelung transplant) managing EoL is a significant and daily part of the service with 73 deaths over the last 5 years (mean: 14.6 per year, range 7-21 per year). However, for smaller CF centres EoL care may be infrequent. Maintaining staff competence is challenging, particularly with such low numbers of deaths per year (UK: $\mathrm{n}=137$ in 2018). ${ }^{2}$

A lack of confidence in initiating EoL discussions can be linked to an absence of adequate, specific training and a reliance on supportive and palliative care teams (SPCTs) taking responsibility for advance care planning (ACP) ${ }^{34}$ Current research suggests that the principal barriers to providing EoL care in $\mathrm{CF}$ are communication, education and coping strategies for patients, their families and clinical teams. ${ }^{5-11}$ 'Burn-out' among clinical teams is a recognised challenge, often attributed to the long-term relationships staff have with patients and the young age of death. ${ }^{8}$ The National Institute for Health and Care Excellence guidelines for CF (2017) recommend that people with $\mathrm{CF}$ and their families are given the opportunity to discuss EoL care with HCP. ${ }^{12}$ No guidelines as to how or when this should be done, or what training clinicians should receive are provided.

European guidelines recommend that all people with $\mathrm{CF}$ are cared for by a full multidisciplinary team (CF-MDT) which comprises of consultants, trainee doctors, clinical nurse specialists, psychologists, pharmacists, dietitians and physiotherapists. ${ }^{13}$ CF-MDTs should work closely with palliative care teams and many centres provide an integrated palliative care model. An online survey was used to evaluate the opinions of the CF-MDT and SPCT working at this centre on the quality of EoL care provided to adults with CF. The results concluded that EoL was not optimal and discussion with patients and family members was too late. Additionally, staff highlighted the lack of structured ACP and limited staff support. These conclusions were reported back to the team as part of an engagement 
exercise. Using information from the survey an improvement project was designed to enhance the quality of EoL care offered to adults with CF through staff education and support. Initially this was in relation to this single $\mathrm{CF}$ centre but as the project progressed, we were able to share our work with other CF centres.

\section{BACKGROUND}

Survival with CF is improving with a current median predicted survival of 47 years; however, CF remains a lifelimiting disease with a median expected age at death of 31 years in the UK in 2018. ${ }^{2}$ Childhood deaths from CF are now rare with 16 deaths of people 19 years and under in the UK in $2018-0.16 \%$ of the UK CF population. ${ }^{2}$ The unpredictable disease trajectory balanced with maintaining hope for a possible life-saving lung transplant and emerging novel therapies, alongside the reality of an inevitable, premature death creates challenges for HCP. ${ }^{3}$

ACP is a voluntary process that can result in the preparation of a documented plan describing individual wishes and thoughts about EoL care. ${ }^{14}$ It has been shown to help families prepare for the death of a loved one, resolve family conflict and help support loved ones with bereavement. ${ }^{1516} \mathrm{ACP}$ is also known to improve EoL care, reduce the number of patients dying in hospital and increase the proportion of patients dying in their preferred place of death. ${ }^{17}$ Although ACP is recommended nationally there is currently no CF-specific ACP document and available documents from other diseases do not meet the needs of individuals with CF. ${ }^{18-21}$ Research shows that most adults with $\mathrm{CF}$ have thought about ACP but clinicians do not initiate discussions, a CF-specific ACP document has been suggested as part of the solution to improve this. ${ }^{19}$

\section{DESIGN}

The project intervention was the development of a 'support package' for staff aiming to help the delivery of high-quality EoL care to adults with CF. The 'support package' includes regular team debriefs following a death, a CF-specific ACP document (with parallel guidance) and a structured, free, online education course available for all HCP. As part of the improvement project key change agents within the CF-MDT were identified and invited to help drive change within the team through their enthusiasm and interest.

Patients and public were not involved in the design and implementation of this quality improvement project, but patients and families were involved in the design of the ACP document and the online EoL course.

\section{MEASUREMENT}

We counted the number of patients who had documented discussions around EoL wishes and recorded if they had died in their preferred location (if documented). We set a target of $75 \%$ of patients with documented EoL discussions and a target of $50 \%$ of patients dying in their preferred location. Most people with CF historically die in hospital due to the complex medical demands at EoL and the unpredictable disease course. Supporting them to die in the community, either at home or in a hospice, is relatively new and comes with many challenges. These include increasing the SPCTs competency and confidence with non-invasive ventilation and education around the role of managing active and palliative therapies in parallel. To measure the success of an educational intervention we asked staff about knowledge and confidence following their engagement in education. We also sought patient feedback throughout the improvement project via surveys.

\section{STRATEGY}

\section{Improvement cycle one}

The aim of the first cycle was to empower staff to initiate conversations around EoL and offer ACP to adults with CF. Barriers to these conversations included staff confidence, lack of experience and fear of upsetting patients. Regular discussion highlighted a lack of understanding about ACP and the differences between discussing prognosis, lung transplant assessment and ACP. Team education and discussions resolved this along with many other questions and concerns. Monthly CF-MDT debriefs were also organised to allow staff not only time to develop positive coping strategies but also reflect and learn from patient deaths.

We set a target of $75 \%$ of patients having EoL discussions and $50 \%$ of patients dying in their preferred location (table 1). After cycle 1, we had not achieved this and there was still a medical/prognostic bias and less documented discussion on symptom control, psychological support, family support and practicalities in terms of getting affairs in order and writing a will.

\section{Improvement cycle 2}

The aim of the second cycle was to introduce a document that could support the CF-MDT to work through all areas of ACP. After reviewing and piloting available (non-CF) ACP documents it became apparent that a disease specific tool was needed, for example, to include elements around transplantation, extra corporeal membrane oxygenation (ECMO), non-invasive ventilation and symptoms. As a result, a CF-specific ACP document was designed. This document was drafted and reviewed by the CF-MDT and SPCT at the Royal Brompton Hospital, and staff from the Royal Marsden Hospital (academic, clinical and research). The document was then adapted following comments and suggestions. Patients, families and bereaved relatives were also invited to codesign and evaluate the document and again comments were reviewed, and the form revised. Lastly, the document was reviewed by seven large UK CF centres and three of the UK CF special interest groups (specialist physiotherapists, clinical psychologists and clinical nurse specialists) before final modifications. 
Table 1 Proportion of patients with documented end of life discussions (ACP), deaths at preferred location and location of death

\begin{tabular}{llll}
\hline & Baseline & Improvement cycle 1 & Improvement cycle 2 \\
\hline $\begin{array}{l}\text { No of patient deaths with documented ACP } \\
\text { discussions (\% of total deaths) }\end{array}$ & $3(10)$ & $13(72)$ & $11(85)$ \\
$\begin{array}{l}\text { No of patients preferred place of death met (\% of } \\
\text { total deaths) }\end{array}$ & $2(7)$ & $8(44)$ & $11(85)$ \\
Location of death & Home=0 & Home=2 & Home=5 \\
& Hospice=2 & Hospice=4 & Hospice=3 \\
& CF centre/ICU=27 & CF centre/ICU=12 & centre/ICU=5 \\
\hline
\end{tabular}

ACP, advance care plan; CF, cystic fibrosis; ICU, intensive care unit.

During the first year patient feedback was captured using a simple survey. Staff using the ACP document undertook formal training including communication skills and guidance on how to complete the document. A guideline document, 'Guidelines for healthcare professions using the ACP', was developed for use alongside the ACP document. The addition of an identifying tab in the electronic patient records allows the ACP documents to be uploaded and made accessible to all members of staff. Patients are given a personal copy (electronic or printed) and are invited to review or change their document whenever they wish.

We predicted that the implementation of the ACP document would increase documented conversations to $75 \%$ and a recorded preferred place of death to $50 \%$ (table 1 ).

\section{Improvement cycle 3}

During the third cycle the ACP document and guidelines for HCP was launched on the UK CF Trust website (national patient charity) and, in the first 18 months, 484 ACPs and 275 guidelines were downloaded from across the UK. To meet the educational needs highlighted in the initial staff survey, an online EoL care course was planned. This course was designed to provide specialist training to CF-MDT members and SPCT professionals and, as it is online, made available internationally.

To support the additional costs of the creation of the online course we received a grant jointly with the UK CF Trust. The course is free for all and accessible via the UK CF Trust website. It is composed of 10 modules led by multiprofessional, international speakers and covers areas including disease trajectory, EoL care, introducing palliative care, symptom control, ACP and place of death. Experiences of a bereaved partner are included, and a patient story is presented via short animations to preserve anonymity. The course was launched in January 2019, to date 258 international HCP (nurses (23\%), doctors $(20 \%)$ and allied health professionals (physiotherapists, dietitians, pharmacists, psychologists, social workers and others) have completed it. International participants are from a range of 26 countries and continents including USA, Australia, New Zealand, East and Western Europe, Russia and Saudi Arabia. The course attracts participants solely through word of mouth. Responses from participants have included the highlighted need for EoL care training, no financial cost and that individuals can flexibly fit the course around busy clinical roles. Individual feedback includes:

'Really well executed and thorough with a range of professions from differing trusts to gain a broad view of different options. "

'Thought provoking and prompted us to review our processes and systems.'

\section{RESULTS}

Additional findings from the improvement cycles are documented here. Following the introduction of the ACP document in improvement cycle 2 , patient feedback was collected. Thirty-three patients (19 female) were offered ACP during the first year. Twenty-six patients $(79 \%)$ completed documentation in full. Nineteen patients completed the survey (table 2). Patient comments following completion of the ACP:

- 'It should always be done by someone who the patient is comfortable talking to and I think it's a very good idea and so do my family'.

- 'I found it enlightening and comprehensive'.

- 'It was very useful'.

- 'Daunting but necessary'.

- 'The form is a really good idea and think it covered everything'.

- 'I found it helpful as it made me think about things that I wouldn't have otherwise thought about writing down'.

- 'I think the form should be introduced to all patients with CF no matter how well or ill as it would have your wishes in place if you were to suddenly become unwell'.

- 'The way it is set out is not so morbid, it's direct but not so harsh you can't answer'.

- 'Very comprehensive and well thought out, no need to change it'.

- 'The timing was right and it was handled sensitively'.

- 'I think it is important that it is a member of the team that you can trust and easily talk to'.

Through the improvement cycles there was an increase in the number of patients who died at home or in a hospice 
Table 2 Patient survey results-following ACP discussion

\begin{tabular}{|c|c|}
\hline Survey question & Results ( $n=19)$ \\
\hline \multirow[t]{2}{*}{ Are you pleased the CF team have asked you about ACP? } & Yes: n=17 (89\%) \\
\hline & No: $n=0$ \\
\hline \multirow[t]{3}{*}{ Do you think ACP was discussed at the right time for you? } & Yes: $n=15(79 \%)$ \\
\hline & Not sure: $n=2(11 \%)$ \\
\hline & Too late: 0 \\
\hline \multirow[t]{2}{*}{ Do you think it is important which member of the team talks to you about ACP? } & Yes: $n=17(89 \%)$ \\
\hline & No: $n=2(11 \%)$ \\
\hline \multirow[t]{5}{*}{ Which team members are best placed to discuss ACP? } & Physiotherapist n=18 (95\%) \\
\hline & CF clinical nurse specialist $n=16(84 \%)$ \\
\hline & Psychologist $n=7$ (37\%) \\
\hline & Ward nurse $n=3$ (16\%) \\
\hline & Dietitian $n=0$ \\
\hline
\end{tabular}

ACP, advance care planning; CF, cystic fibrosis.

(table 1). These patients were all supported with early community palliative care referrals and proactive planning. Patients on an active lung transplant list often died in hospital as many were appropriate for intensive care admission and ECMO as a bridge to transplant. As part of the ACP process, discussions around preferred place of death and different possible scenarios for the individual patient are always completed.

\section{Lessons and limitations}

The engagement of the CF-MDT was key to changing the team culture around EoL care. Using a staff survey and presenting the data back allowed open discussion around how to improve care. This was undoubtedly due to a combination of unanimous identification of need and patient involvement. Comments from the CF-MDT included: 'We need more emphasis on EoL care as a whole CF team' and 'Our issue is inconsistency of practice-some patients will get excellent EoL care and others inadequate'.

Although requested by the CF-MDT, the debrief sessions ran into a few early barriers, this was because they were initially organised reactively following a death with not enough notice for all staff to attend. This issue was addressed by prebooking the debrief sessions every 2 months and asking individual team members to lead sessions allowing the team to choose the direction of discussion and breakdown traditional medical hierarchy. As with any busy clinical team, finding time when the majority of the CF-MDT could attend was difficult and involving the ward nurses working shifts was particularly challenging. We found bimonthly meetings on the same day and time effective for the CF-MDT but were unable to find a solution to fully including ward nursing staff. Currently, the ward nursing team complete small nursing team debriefs and then send representation to the debrief with feedback from the wider nursing team.

At times individuals in the CF-MDT struggled to have honest discussions about EoL care for fear of looking like they were giving up. Overcoming this cultural barrier required engagement from everyone. Using the staff survey enabled the team to see this was a key area for quality improvement within the service. Although education and debriefs resulted in improvements the ACP has allowed sustained changes which would have been hard to achieve long term without it. The ACP document received positive patient feedback and supports current literature that patients want time and opportunity to talk about their EoL care and consider their options. ${ }^{18}$ Patients were comfortable talking about death and EoL care which is again supported by other authors. ${ }^{18}$ Sawicki et al suggested that ACP rates would be low in a CF population as culturally discussing ACP is not experienced by young people; however, this was not seen in our cohort with a mean age of 37 years, with patients as young as 17 years. Low rates of ACP have been recorded in CF and post-transplant populations and this maybe because patients are not being offered the opportunity and will not, or feel too inhibited to initiate the conversation, even though they are comfortable talking about death when offered the chance. ${ }^{182}$ Similar initiatives to increase ACP in other populations have succeeded by also advocating direct professional to patient communication. ${ }^{23-25}$

This project led to an increase in the number of patients dying at their preferred location, including more 
patients dying at home or in a hospice. Traditionally most people with CF die in hospital, however, this project has highlighted the need to have proactive discussions as part of ACP to ascertain what each individual patient's preference would be. Some patients decided their preferred location of death was hospital in a familiar surrounding with staff they had often known for years, however, others wanted to be at home or in the less clinical environment of a hospice. Facilitating conversation on place of death early enabled patients to engage with community palliative care teams early, visit hospices and discuss the options with their loved ones. CF has an uncertain disease trajectory making it even more essential for clinical teams to initiate early discussions around preferred location of death.

Patients want to complete the ACP discussion and document with a member of staff they know well. Patients identified the physiotherapists and clinical nurse specialists as team members they felt were best placed to complete ACP. We therefore now have a small group of physiotherapists and nurses who are very competent and confident at completing ACP. We need to continue to ensure these skills are disseminated across the wider CF-MDT to meet all patient needs. Patients likely identify physiotherapists and nurse specialists as best placed to complete ACP because of familiarity. In our centre, patients usually spend the most amount of time with these professions and build close relationships over many years. We acknowledge that CF centres may vary in terms of roles and responsibilities and therefore the most appropriate staff to complete ACP may well differ between centres.

Adequate education and support for staff undertaking advance care discussions is essential to ensure coping and limit 'burnout'. Education in this instance needs to focus on breaking down the barriers between professionals initiating advance care conversations and the patient. Research shows that patients will react in very different ways to being offered ACP and may experience a degree of emotional distress appropriate for the situation, however, most will accept the offer of a discussion and find it empowering. ${ }^{1526}$

We acknowledge that this project was led from a single CF centre and other centres may have very different challenges, especially in terms of culture. However, when seeking the involvement of other centres in designing the ACP no other centres consistently completed ACP with a standardised document. CF centres with high staff turnover may need to focus on education and support, however, using this national document will allow staff to move between centres and feel confident in using the ACP documentation. The high number of ACP downloads across the UK and variety of HCP completing the online course highlights this project has impacted more than a single CF centre. To continue to change culture in the CF community we need to keep engaging patients and their loved ones and ensuring $\mathrm{CF}$ teams continue to receive regular education and support. The online course will require regular updating and promotion which we intend to complete at international CF meetings, through other CF education events and via word of mouth.

A limitation of the online course is that there is no formal assessment of the change in learners' knowledge. We did seek self-reported feedback, but it lacked objectivity. In the future a simple knowledge questionnaire could be added to the start and end of the online course to collect this information. All feedback on the course was positive with many commenting on increased confidence and knowledge and reassessing the EoL care their service provided.

Thankfully childhood deaths from CF are low in the UK, however, this likely results in an even greater need for education and support for paediatric staff who may be less used to supporting patients at the EoL. Dissemination of our learning to other CF centres both nationally and internationally will need to include paediatric as well as adult centres.

The biggest lessons learnt from this project are around staff engagement and ensuring this is established before starting the project. Additionally, relatively high staff turnover means that new starters need to be educated to allow ACP to be part of everyone's role. All new staff complete the online course and have ACP teaching from a senior member of the team which includes observing ACP discussions. Although we saw a direct impact on patient care and measured staff feedback on an informal basis, it would have been good to measure staff confidence throughout the improvement cycles to ensure staff were feeling supported and engaged in the process.

\section{CONCLUSION}

The aim of this project was to improve the quality of EoL care through staff education and support, with the intention to empower staff to start early conversations around EoL thereby better meeting patient wishes. During the project, we successfully integrated bimonthly team debriefs which have resulted in team learning and support. We also designed a CF-specific ACP that is now available on the UK CF Trust website and a free, internationally available, online training course for all HCP. We found that by offering staff support and training alongside empowering them with a structed ACP document we could positively change the culture of the CF-MDT resulting in early ACP and more patients dying in their preferred location.

Although we acknowledge that this is an ongoing process, the adult CF service at this centre now uses formal documentation for ACP which is accessible to the entire team. ACP is introduced in a timely manner by staff trained to support the process and each document can be reviewed regularly, either by the CF-MDT or at the request of the individual patient. Final wishes are known and supported by the team and patients are encouraged to share the ACP with loved ones. Recent literature suggests that CF teams, although identifying the issue, currently struggle to find solutions. Thanks to the enthusiasm of 
HCP working in partnership with patients we can suggest a model of care that may work in other adult CF centres. It is important, however, that the ACP document does not become a 'tick box exercise' and should not alone define ACP, instead the document should form a structure to empower staff to assist patients in documenting their thoughts and wishes.

Twitter Fiona Cathcart @fionacathcart1

Acknowledgements We would like to thank our patients, their relatives and other CF specialist centres involved in reviewing and developing the ACP document. We also acknowledge the support of the CF MDT at the Royal Brompton Hospital and the CF Trust.

Contributors FC led the day to day project and wrote the first draft. SM has provided clinical leadership throughout the project and assisted with redrafting the paper. JW also provided clinical leadership and support with the project and supported the writing up phase.

Funding The Sam and Bella Sebba Charitable Trust awarded jointly with the Cystic Fibrosis Trust.

Competing interests None declared.

Patient and public involvement Patients and/or the public were not involved in the design, or conduct, or reporting, or dissemination plans of this research.

Patient consent for publication Not required.

Provenance and peer review Not commissioned; externally peer reviewed.

Data availability statement Data are available upon request.

Open access This is an open access article distributed in accordance with the Creative Commons Attribution Non Commercial (CC BY-NC 4.0) license, which permits others to distribute, remix, adapt, build upon this work non-commercially, and license their derivative works on different terms, provided the original work is properly cited, appropriate credit is given, any changes made indicated, and the use is non-commercial. See: http://creativecommons.org/licenses/by-nc/4.0/.

ORCID iD

Fiona Cathcart http://orcid.org/0000-0001-5517-9317

\section{REFERENCES}

1 Burgel P-R, Bellis G, Olesen HV, et al. Future trends in cystic fibrosis demography in 34 European countries. Eur Respir J 2015;46:133-41.

2 UK Cystic Fibrosis Trust. Uk cystic fibrosis registry 2018 annual data report. UK; 2019.

3 Robinson WM. Palliative and end-of-life care in cystic fibrosis: what we know and what we need to know. Curr Opin Pulm Med 2009;15:621-5.

4 Madge S, Sands D. End of life: have we got it right? J Cyst Fibros 2016:15:2-3.

5 Sands D, Repetto T, Dupont LJ, et al. End of life care for patients with cystic fibrosis. J Cyst Fibros 2011;10 Suppl 2:S37-44.
6 Clisby N, Shaw S, Cormack M. Psychological impact of working with patients with cystic fibrosis at end-of-life, pre-transplant stage. Palliat Support Care 2013;11:111-21.

7 Lowton K. 'A bed in the middle of nowhere': parents' meanings of place of death for adults with cystic fibrosis. Soc Sci Med 2009;69:1056-62.

8 Braithwaite M, Philip J, Tranberg H, et al. End of life care in CF: patients, families and staff experiences and unmet needs. J Cyst Fibros 2011;10:253-7.

9 Chapman E, Landy A, Lyon A, et al. End of life care for adult cystic fibrosis patients: facilitating a good enough death. $J$ Cyst Fibros 2005;4:249-57.

10 Mitchell I, Nakielna E, Tullis E, et al. Cystic fibrosis. end-stage care in Canada. Chest 2000;118:80-4.

11 Goggin J, Cohen RI. Cf healthcare workers feel unprepared in providing suitable end of life care and desire more education: results of a nationwide survey. J Cyst Fibros 2016;15:85-9.

12 National Institute for Clinical Excellence (NICE). Cystic fibrosis: diagnosis and management. NICE Guidleine [NG78], 2017.

13 Smyth AR, Bell SC, Bojcin S, et al. European cystic fibrosis Society standards of care: best practice guidelines. J Cyst Fibros 2014;13 Suppl 1:S23-42.

14 Mullick A, Martin J, Sallnow L. An introduction to advance care planning in practice. BMJ 2013;347:f6064.

15 Rhee JJ, Zwar NA, Kemp LA. Advance care planning and interpersonal relationships: a two-way street. Fam Pract 2013;30:219-26.

16 Detering KM, Hancock AD, Reade MC, et al. The impact of advance care planning on end of life care in elderly patients: randomised controlled trial. BMJ 2010;340:c1345.

17 Temel JS, Greer JA, Muzikansky A, et al. Early palliative care for patients with metastatic Non-Small-Cell lung cancer. N Engl J Med Overseas Ed 2010;363:733-42.

18 Kazmerski TM, Weiner DJ, Matisko J, et al. Advance care planning in adolescents with cystic fibrosis: a quality improvement project. Pediatr Pulmonol 2016;51:1304-10.

19 Sawicki GS, Dill EJ, Asher D, et al. Advance care planning in adults with cystic fibrosis. J Palliat Med 2008;11:1135-41.

20 Fried TR, Bullock K, lannone L, et al. Understanding advance care planning as a process of health behavior change. J Am Geriatr Soc 2009;57:1547-55.

21 Dellon EP, Chen E, Goggin J, et al. Advance care planning in cystic fibrosis: current practices, challenges, and opportunities. J Cyst Fibros 2016;15:96-101.

22 Song M-K, De Vito Dabbs AJ. Advance care planning after lung transplantation: a case of missed opportunities. Prog Transplant 2006;16:222-5.

23 Ramsaroop SD, Reid MC, Adelman RD. Completing an advance directive in the primary care setting: what do we need for success? $J$ Am Geriatr Soc 2007;55:277-83.

24 Brown JB, Beck A, Boles M, et al. Practical methods to increase use of advance medical directives. J Gen Intern Med 1999;14:21-6.

25 Heffner JE, Fahy B, Hilling L, et al. Outcomes of advance directive education of pulmonary rehabilitation patients. Am J Respir Crit Care Med 1997;155:1055-9.

26 Curtis JR, Engelberg R, Young JP, et al. An approach to understanding the interaction of hope and desire for explicit prognostic information among individuals with severe chronic obstructive pulmonary disease or advanced cancer. J Palliat Med 2008;11:610-20. 